\title{
Blood Pressure Variability and Outcomes in End-Stage Renal Disease Patients on Dialysis: A Systematic Review and Meta-Analysis
}

\author{
Huihui Li ${ }^{a}$ Jing Xue ${ }^{b}$ Wenjie Dai ${ }^{c}$ Xiaohua Liao ${ }^{a}$ Peng Zhu ${ }^{a}$ \\ Qiaoling Zhou ${ }^{\mathrm{a}}$ Wenhang Chen ${ }^{\mathrm{a}}$ \\ a Department of Nephrology, Xiangya Hospital, Central South University, Changsha, China; \\ bepartment of Scientific Research, Xiangya Hospital, Central South University, \\ Changsha, China; ' Xiangya School of Public Health, Central South University, Changsha, China
}

\section{Keywords}

Blood pressure variability $\cdot$ End-stage renal disease $\cdot$ Hemodialysis $\cdot$ Systematic review .

Meta-analysis

\begin{abstract}
Objective: Previous studies have suggested that blood pressure variability (BPV) is associated with an increased risk of mortality and cardiovascular events in patients on dialysis. However, the results are inconsistent. A comprehensive literature review was conducted to analyze the association between BPV and outcomes in patients on dialysis. Methods: Articles in Embase, Medline, and Web of Science from the date of inception through January 1, 2020, were identified. The outcomes were all-cause and cardiovascular mortality and cardiovascular events. The risk of bias was assessed using the Newcastle-Ottawa scale tool. Random effects models were used to pool the overall effect sizes. Two reviewers extracted the data independently. Meta-regression and subgroup analyses were performed to explore potential heterogeneity. Results: Fifteen eligible studies were included, and all enrolled hemodialysis recipients only. The overall risk of bias for the included studies was low. A 1-SD increase in systolic BPV was associated with higher risks of all-cause mortality ( $\left.\mathrm{HR}=1.18 ; 95 \% \mathrm{Cl} 1.11-1.26, I^{2}=53.8 \%\right)$, cardiovascular mortality ( $\left.\mathrm{HR}=1.23 ; 95 \% \mathrm{Cl} 1.10-1.37, R^{2}=57.2 \%\right)$, and cardiovascular events $\left(\mathrm{HR}=1.27 ; 95 \% \mathrm{Cl} 1.07-1.51, \mathrm{I}^{2}=69.3 \%\right)$. Likewise, a 1-SD increase in diastolic BPV was associated with higher $\mathrm{HR}$ for all-cause and cardiovascular mortality $(\mathrm{HR}=1.14 ; 95 \% \mathrm{Cl} 1.05-1.23$, $R^{2}=0.0 \%$, and $\mathrm{HR}=1.14 ; 95 \% \mathrm{Cl} 0.94-1.38, R^{2}=0.0 \%$, respectively). Conclusions: $\mathrm{A}$ greater
\end{abstract}

H.L. and J.X. contributed equally to this work. 


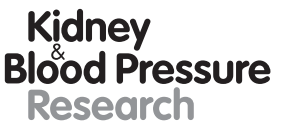

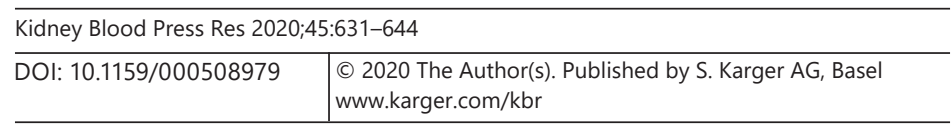

Li et al.: BPV Predicts Adverse Outcomes in Patients on Dialysis

BPV is associated with higher risks of cardiovascular and mortality outcomes in patients on hemodialysis. Further research is required to determine whether BPV may be useful either as a marker enabling individualized treatment of cardiovascular risk or as a treatment target in its own right.

(C) 2020 The Author(s)

Published by S. Karger AG, Basel

\section{Introduction}

Despite technical advances in hemodialysis (HD) and peritoneal dialysis (PD), patients on dialysis have substantially higher risks of cardiovascular events and mortality rates than the general population [1-4]. The prevalence of hypertension is high in those with end-stage renal disease (ESRD) receiving dialysis, and it is a prominent risk factor for cardiovascular disease morbidity and mortality and contributes to heart failure and arrhythmias [5]. Accurate, appropriate blood pressure (BP) measurement is vital. As in the general population, treatment of hypertension may have a significant impact on the survival of patients receiving dialysis [6, 7].

Mean BP might not fully describe the relationship between BP and cardiovascular disease or mortality. BP variability (BPV) describes the extent of BP fluctuations over time. Based on the time interval of BP measurements, BPV is classified into long- and short-term BPV. Longterm BPV usually refers to visit-to-visit BPV, whereas short-term BPV is usually measured by ambulatory BP monitoring during a 24- to 48-h period [8]. Various factors, such as age, increased vascular stiffness, sympathetic nervous system activity, seasonal changes, and treatment-related factors, may influence BP fluctuations [9]. Growing evidence suggests that BPV is linked to greater risks of heart disease and incident chronic kidney disease (CKD) and stroke, independently of the effects of the mean BP, in the general population, as well as in patients with hypertension or diabetes [10-13].

Patients with CKD have increased BPV [14]. Patients undergoing HD regularly have fluids removed for short periods of time and are prone to greater BPV, which may contribute to their burden of cardiovascular disease [14]. Studies have found an association between the mean BP and outcomes in patients on HD [15-17]. Over the last decade, more studies have explored the impact of BPV on the prognosis of HD patients, with inconsistent results [18-25]. Therefore, we conducted a systematic review and meta-analysis to assess the prognostic significance of BPV on cardiovascular and all-cause mortality and cardiovascular events in ESRD patients on dialysis.

\section{Methods}

\section{Search Strategy}

This study was conducted in accordance with Meta-Analysis of Observational Studies in Epidemiology (MOOSE) guidelines [26]. The methodology complied with the Cochrane Handbook for Interventional Systematic Reviews [27]. This article was written in a manner that adhered to the Preferred Reporting Items for Systematic Reviews and Meta-Analyses (PRISMA) statement [28]. Two reviewers (H.L. and J.X.) independently searched Medline (for studies published from January 1, 1946, to January 1, 2020), Embase (from January 1, 1960, to January 1, 2020), and Web of Science (from the date of inception to January 1, 2020) for eligible articles in English or Chinese. The search strategy was devised by an experienced librarian at Central South University and is available in the supplementary material (for all online suppl. material, see www.karger.com/doi/10.1159/000508979). The following search 


\section{Kidney \\ Blood Pressure \\ Research}

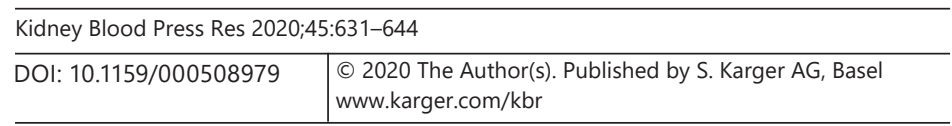

Li et al.: BPV Predicts Adverse Outcomes in Patients on Dialysis

terms were used: "blood pressure variability," "hemodialysis," "peritoneal dialysis," and other comparable search terms. We also searched the reference lists of relevant articles manually for potential additional studies.

\section{Inclusion and Exclusion Criteria}

The included studies were required to meet the following criteria: (1) cohort design studies, including prospective or retrospective cohorts; (2) population of ESRD patients on either HD or PD; (3) BPV was monitored; and (4) at least 1 of the following outcomes was reported: all-cause mortality, cardiovascular mortality, and cardiovascular events (including coronary heart disease, myocardial infarction, heart failure, and stroke).

Studies were excluded if they were a review, a supplement, a commentary article, an editorial, or a conference article. If more than 1 study reported on an overlapping population, the study with the shorter follow-up duration or the smaller population was excluded. Discrepancies were resolved by discussion.

\section{Data Extraction}

Two independent investigators extracted information, including the study characteristics (first author's name, publication year, country, database, study design, follow-up duration, sample size, age, and sex), exposure assessment (BP measurement and BPV metrics), outcome assessment (all-cause mortality, cardiovascular mortality, and cardiovascular events), and association estimates (most fully adjusted HR and 95\% CI for the corresponding category), using prespecified forms. A third investigator (W.C.) was consulted in the case of disagreement.

\section{Assessment of Study Quality}

The risk of bias in the study design was assessed using the Newcastle-Ottawa scale for cohort studies [29]. Specifically, studies were assessed using a quality score in the following 3 domains: selection of the study groups (maximum 4 points), group comparability (maximum 2 points), and ascertainment of outcome in the cohorts (maximum 3 points). A higher score indicated better quality.

\section{Data Synthesis and Statistical Analysis}

All statistical analyses were conducted using Stata version 12 (Stata, College Station, TX, USA). The included studies examined BPV in multiple metrics. The BPV metrics included in the meta-analyses were considered in the following order: coefficient of variation (CV), SD, variability independent of the mean (VIM), average real variability (ARV), and residual variability. To allow quantitative synthesis of estimates across studies, we pooled HR for a 1-SD change to measure the effect of the association between BPV and outcomes using random effects models. When HR indicated specific BPV increment intervals (e.g., 1 or $10 \mathrm{~mm} \mathrm{Hg}$ ), HR for the 1-SD increment were calculated with the corresponding root of the original value. For instance, if the reported HR for a 1-mm Hg increment were 1.20, and the SD of the BPV were $3.0 \mathrm{~mm} \mathrm{Hg}$, then the HR per SD increment change would be the third power of 1.20 (i.e., 1.73) $[30,31]$. When HR were reported for quintiles, quantiles, or tertiles of the BPV, the HR for a 1-SD increment were estimated as 1/2.80 times the log HR to compare the top and bottom quintiles, $1 / 2.54$ times the log HR to compare the top and bottom quartiles, and 1/2.18 times the log HR to compare the top and bottom tertiles [32]. Between-study heterogeneity was assessed using Cochrane $Q$ and $I^{2}$ statistics, and a $p$ value $<0.10$ or $I^{2}>50 \%$ was considered heterogeneous [33]. Meta-regression and subgroup analyses were used to explore the sources of heterogeneity in our main finding. In addition, to determine the influence of individual studies on the overall effect, we performed a sensitivity analysis by repeating the meta- 


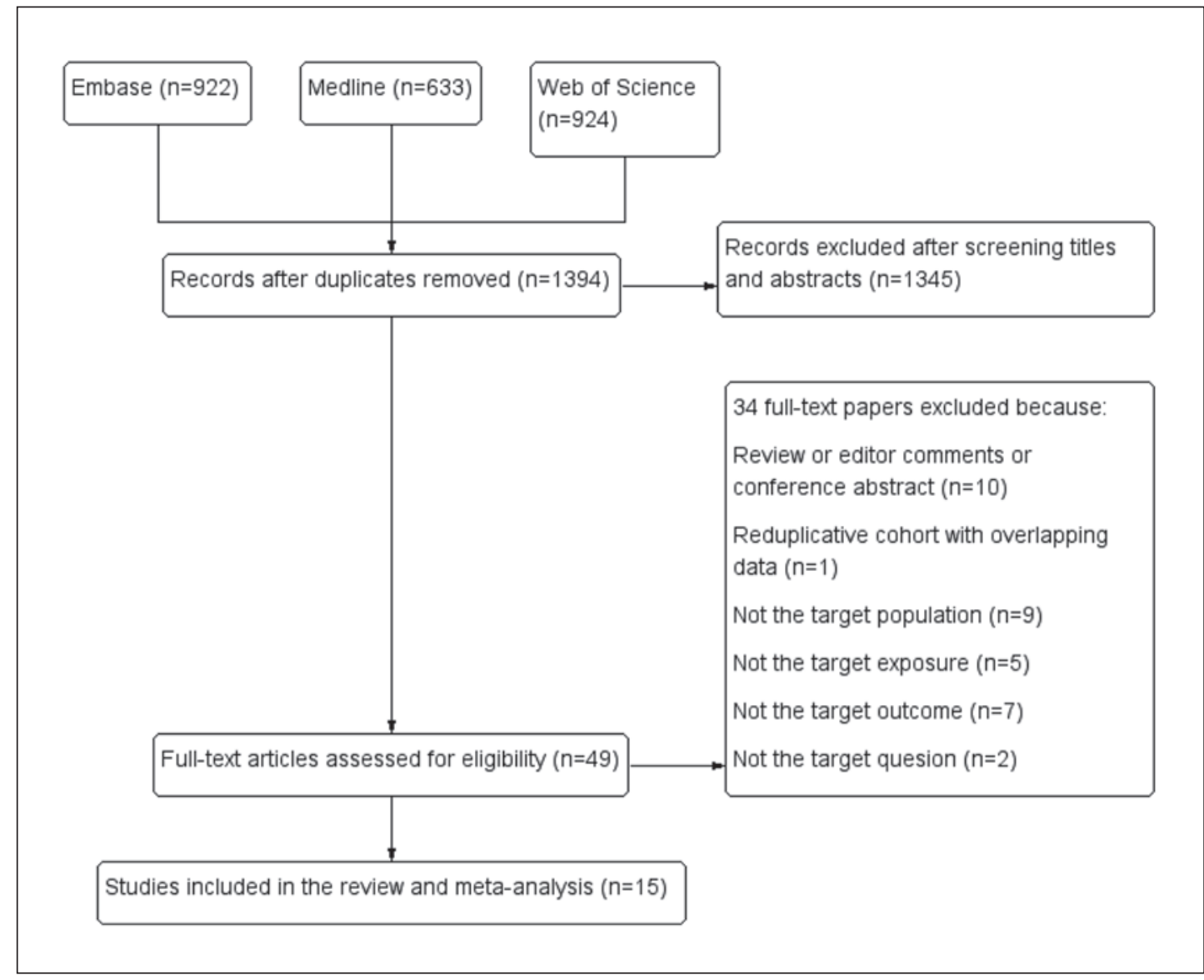

Fig. 1. Flowchart of the literature search.

analysis and removing one study at a time. Publication bias was assessed using the Begg test and presented graphically with funnel plots of precision [34]. Two-sided $p<0.05$ was considered statistically significant unless otherwise specified.

\section{Results}

\section{Characteristics of the Included Studies}

Figure 1 summarizes the results of the literature search, which yielded 1,394 citations after deduplication, 1,345 of which were excluded after title and abstract review. The full texts of the remaining 49 articles were reviewed, and 34 were excluded because the population, exposure, or outcomes were not of interest or the article was a review, editor's comment, or reference abstract. One study presented overlapping data using the same datasets and was excluded [19]. Ultimately, the review included 15 eligible studies [18, $20-25,35-42]$. Results from 2 studies did not provide sufficient data for the pooled analysis $[22,42]$. Thus a total of 13 studies were included in the meta-analyses $[18,20,21,23-25$, 35-41].

The characteristics of the included studies are summarized in Table 1. The review included 31,841 patients on HD; no PD patients were included. The follow-up ranged from 0.5 to 14 years. Of the included studies, 7 were conducted in Asia (4 in China, 2 in Japan, and 


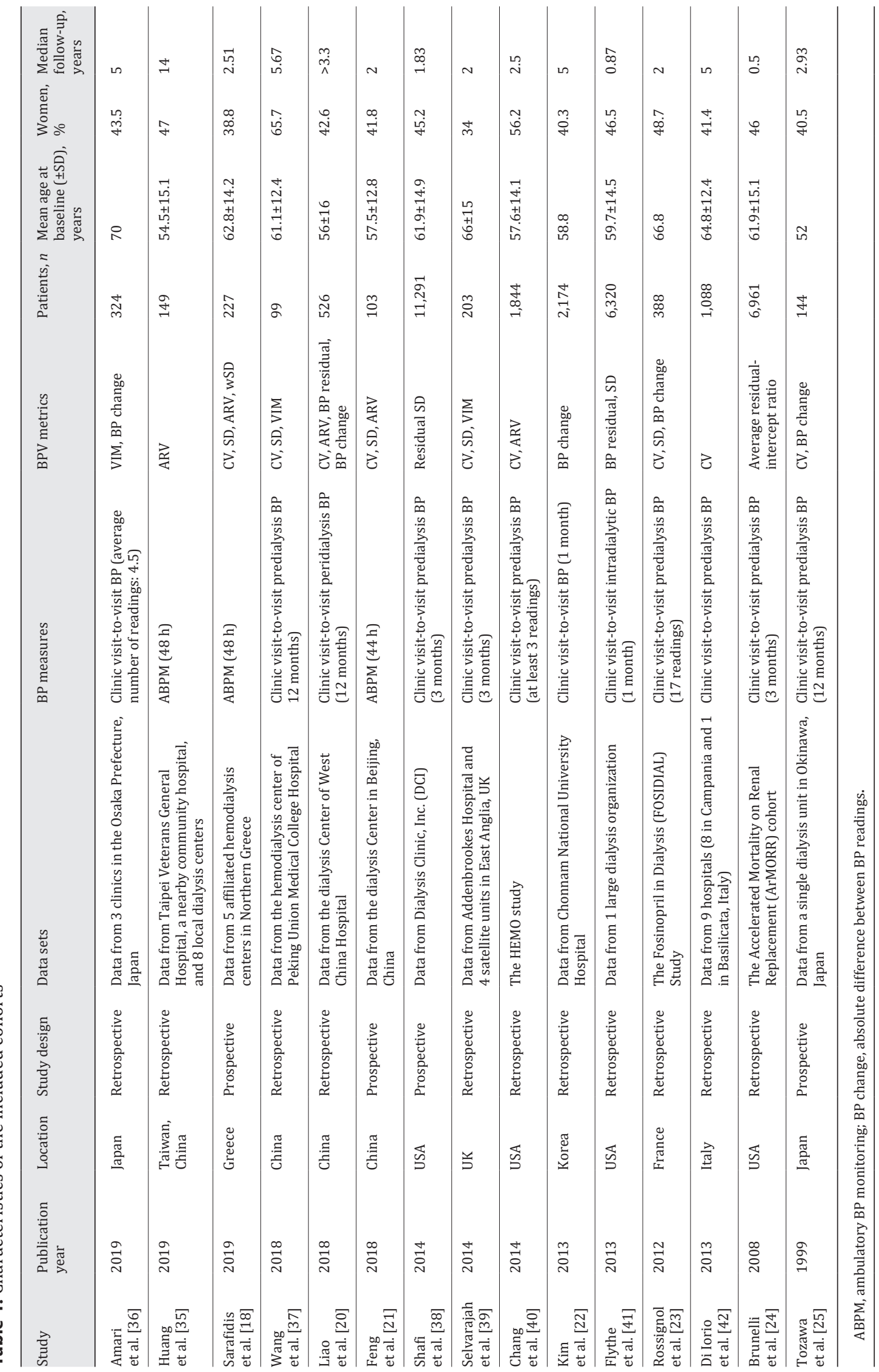




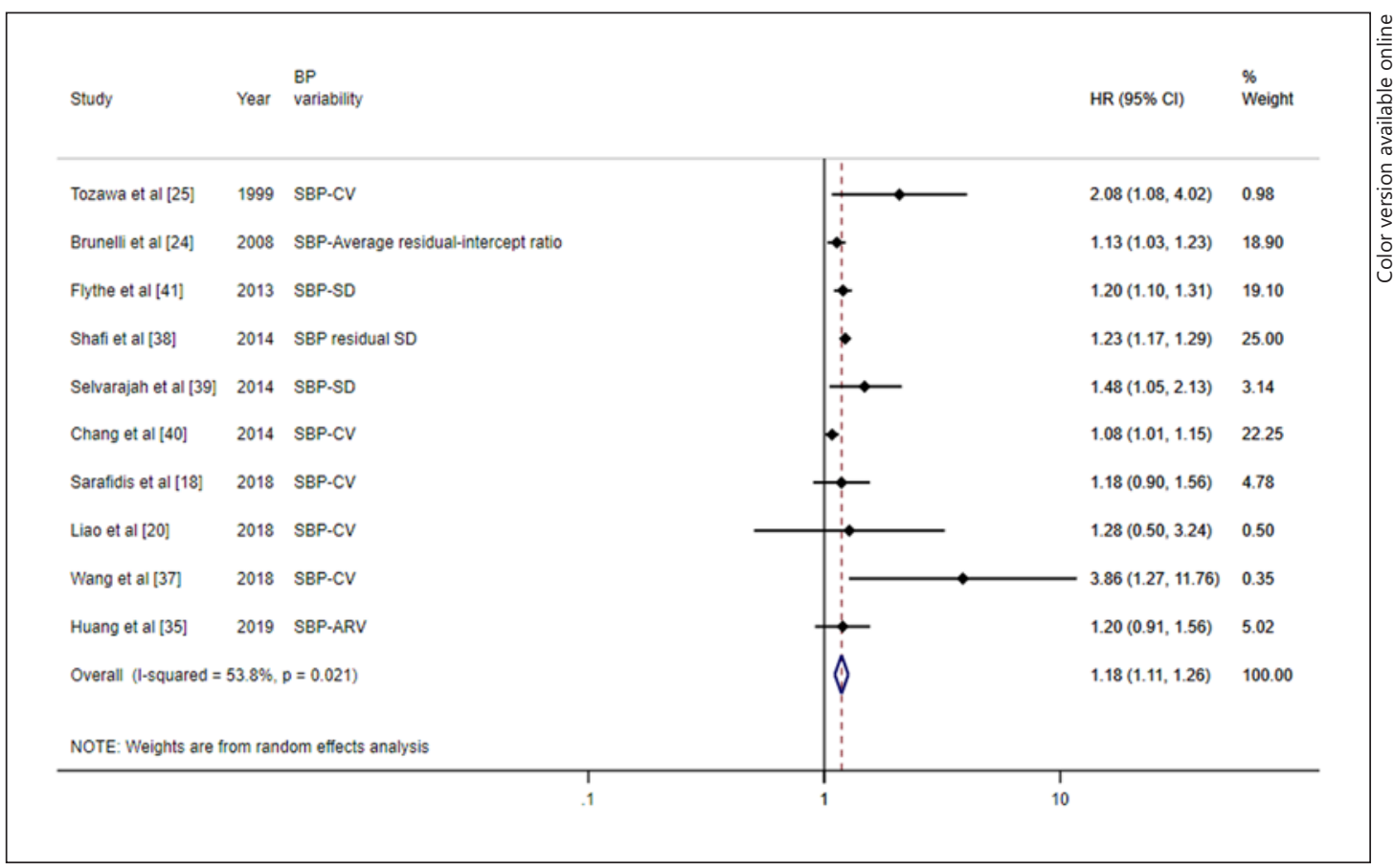

Fig. 2. Forest plot of the relationship between a 1-SD increment in SBPV and all-cause mortality. Boxes represent $\mathrm{HR}$ and lines represent $95 \% \mathrm{CI}$ for individual studies. The diamonds and their width represent pooled HR and 95\% CI, respectively. SBP, systolic BP.

1 in Korea) and the remaining 8 were conducted in North America or Europe (4 in the USA, 1 in France, 1 in Greece, 1 in Italy, and 1 in the UK). All of the studies reported adjusted estimates. The overall quality of the included studies was high according to the Newcastle-Ottawa scale assessment tool (online suppl. Table S1).

\section{Association between Systolic BPV and Outcomes}

Ten studies were included in the analysis of the association between systolic BPV (SBPV) and all-cause mortality. A 1-SD increase in SBPV was associated with an 18\% higher risk of all-cause mortality in HD patients (HR $=1.18$; 95\% CI 1.11-1.26, $I^{2}=53.8 \%$; Fig. 2). The heterogeneity was moderate. Nine studies described the association between each SD increment in SBPV and cardiovascular mortality. The HR for cardiovascular mortality associated with each 1-SD increase in SBPV was 1.23 (95\% CI 1.10-1.37, $I^{2}=57.2 \%$; Fig. 3). For cardiovascular events, the meta-analysis included 4 studies. The HR associated with each 1-SD increase in SBPV was 1.27 (95\% CI 1.07-1.51, $I^{2}=69.3 \%$; Fig. 4).

\section{Association between Diastolic BPV and Outcomes}

Three studies reported an association between diastolic BPV (DBPV) and all-cause mortality. A 1-SD increase in DBPV was associated with a 14\% higher risk of all-cause mortality in HD patients (HR $=1.14 ; 95 \%$ CI 1.05-1.23, $I^{2}=0.00 \%$; Fig. 5). Two studies described an association between each SD increment in DBPV and cardiovascular mortality. The HR for cardiovascular mortality associated with a 1-SD increase in DBPV was 1.14 (95\% CI 0.94$1.38, I^{2}=0.00 \%$; Fig. 6 ). One study reported a relationship between cardiovascular events and DBPV [18]. The HR was 1.35 (95\% CI 1.02-1.79) with a 1-SD increase in DBPV for cardiovascular events. 


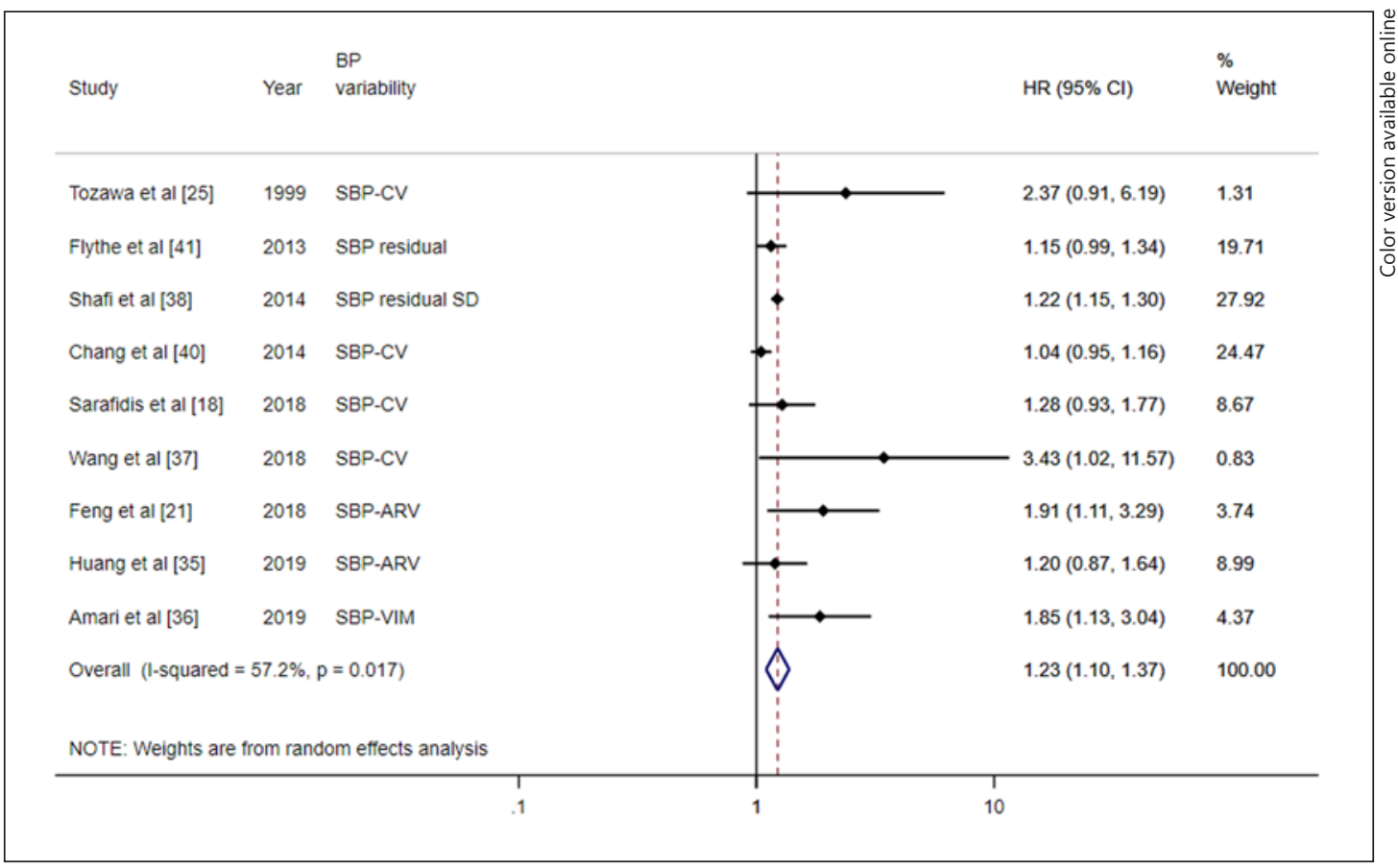

Fig. 3. Forest plot of the relationship between a 1-SD increment in SBPV and cardiovascular mortality. Boxes represent HR and lines represent 95\% CI for individual studies. The diamonds and their width represent pooled HR and 95\% CI, respectively. SBP, systolic BP.

\section{Association between Long-Term BPV and Outcomes}

Eight studies examined long-term SBPV and all-cause mortality. A 1-SD increase in longterm SBPV was associated with a $19 \%$ higher risk of all-cause mortality $(\mathrm{HR}=1.19 ; 95 \% \mathrm{CI}$ 1.10-1.28, $I^{2}=64.0 \%$; online suppl. Fig. S1). Six studies assessing long-term SBPV and cardiovascular mortality showed a significant relation (HR per 1 -SD increase $=1.20$; 95\% CI $1.06-$ $1.37, I^{2}=67.6 \%$; online suppl. Fig. S2). Three studies examining long-term SBPV and cardiovascular events were included in the meta-analysis (HR per 1 -SD increase $=1.20 ; 95 \% \mathrm{CI}$ $1.02-1.41, I^{2}=61.1 \%$; online suppl. Fig. S3). Only 1 study provided sufficient data assessing long-term DBPV and all-cause mortality (HR per 1-SD increase $=1.15 ; 95 \%$ CI 1.06-1.26) [24]. No study reported the relation between a 1-SD increase in long-term DBPV and cardiovascular mortality and cardiovascular events.

\section{Association between Short-Term BPV and Outcomes}

Two studies examined short-term SBPV and all-cause mortality. The HR for all-cause mortality associated with a 1-SD increase in SBPV was 1.19 (95\% CI 0.98-1.44, $I^{2}=0.00 \%$; online suppl. Fig. S4). Three studies examined short-term SBPV and cardiovascular mortality. The HR for a 1-SD increase in short-term SBPV and cardiovascular mortality was 1.32 (95\% CI 1.06-1.65, $I^{2}=8.6 \%$; online suppl. Fig. S5). One study provided sufficient data assessing short-term SBPV and cardiovascular events (HR per 1-SD increase $=1.52 ; 95 \%$ CI 1.14-2.04) [18].

Two studies examining long-term DBPV and all-cause mortality were included in the meta-analysis (HR per 1-SD increase $=1.09$; 95\% CI 0.91-1.30, $I^{2}=0.00 \%$; online suppl. Fig. S6). Two studies examined short-term DBPV and cardiovascular mortality. The HR for cardiovascular mortality associated with a 1-SD increase in DBPV was $1.14\left(95 \%\right.$ CI $0.94-1.38, I^{2}=$ 


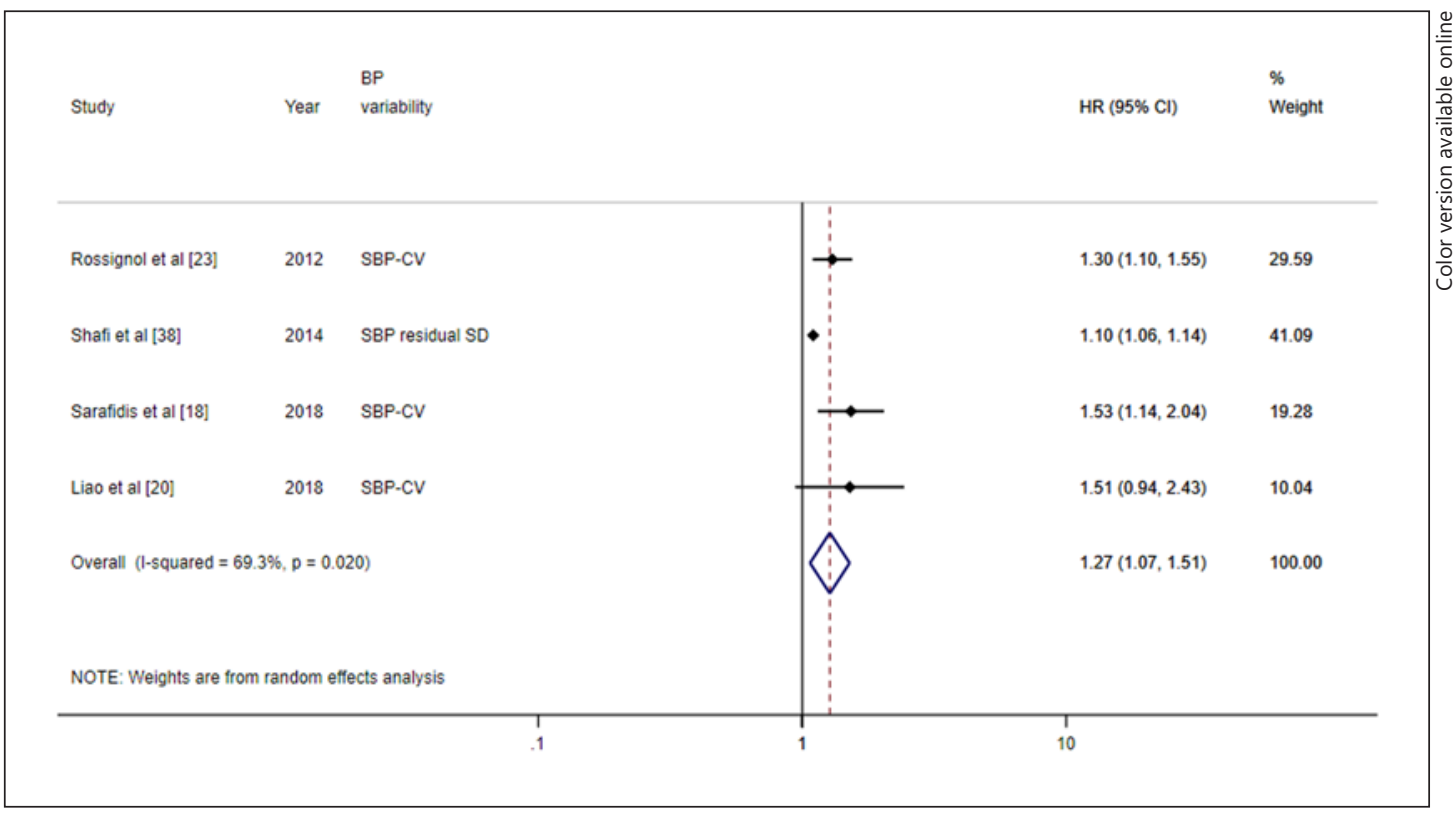

Fig. 4. Forest plot of the relationship between a 1-SD increment in SBPV and cardiovascular events. Boxes represent HR and lines represent $95 \% \mathrm{CI}$ for individual studies. The diamonds and their width represent pooled HR and the $95 \% \mathrm{CI}$, respectively. SBP, systolic BP.

0.00\%; online suppl. Fig. S7). One study provided sufficient data assessing short-term DBPV and cardiovascular events (HR per 1-SD increase $=1.35$; 95\% CI 1.02-1.79) [18].

\section{Meta-Regression and Subgroup Analyses}

Moderate heterogeneity was seen in the meta-analysis of the association between SBPV and outcomes. To investigate the impact of the study characteristics on the study estimates of HR in the analysis of SBPV on all-cause mortality, we performed a meta-regression analysis on the study region (Asia vs. the others, $p=0.30$ ), study design (prospective and retrospective, $p=0.27$ ), age (mean age $>60$ and $\leq 60$ years, $p=0.51$ ), sex ( $>50$ and $\leq 50 \%$ women, $p=0.06$ ), follow-up duration ( $p=0.84$ ), and adjustment factors (adjusted or not adjusted for interdialytic weight gain, $p=0.61$ ). The meta-regression did not reveal a significant source of heterogeneity. The results of different subgroup analyses are shown in Table 2. The HR values were not significant in the subgroup analyses of CV, ARV, and groups with $>50 \%$ women. The other subgroups remained significant.

\section{Bias and Sensitivity Analysis}

Among the studies, there was no evidence of publication bias according to the Begg test (Begg test $z=1.07, p>0.228$; online suppl. Fig. S8). The results remained consistent during sensitivity analysis, indicating that the meta-analysis was stable (online suppl. Fig. S9).

\section{Discussion and Conclusion}

This study found that a greater BPV was associated with higher risks of all-cause mortality, cardiovascular-related mortality, and cardiovascular events in patients on regular HD. Both SBPV and DBPV were strong predictors of adverse outcomes. To our knowledge, this is the 


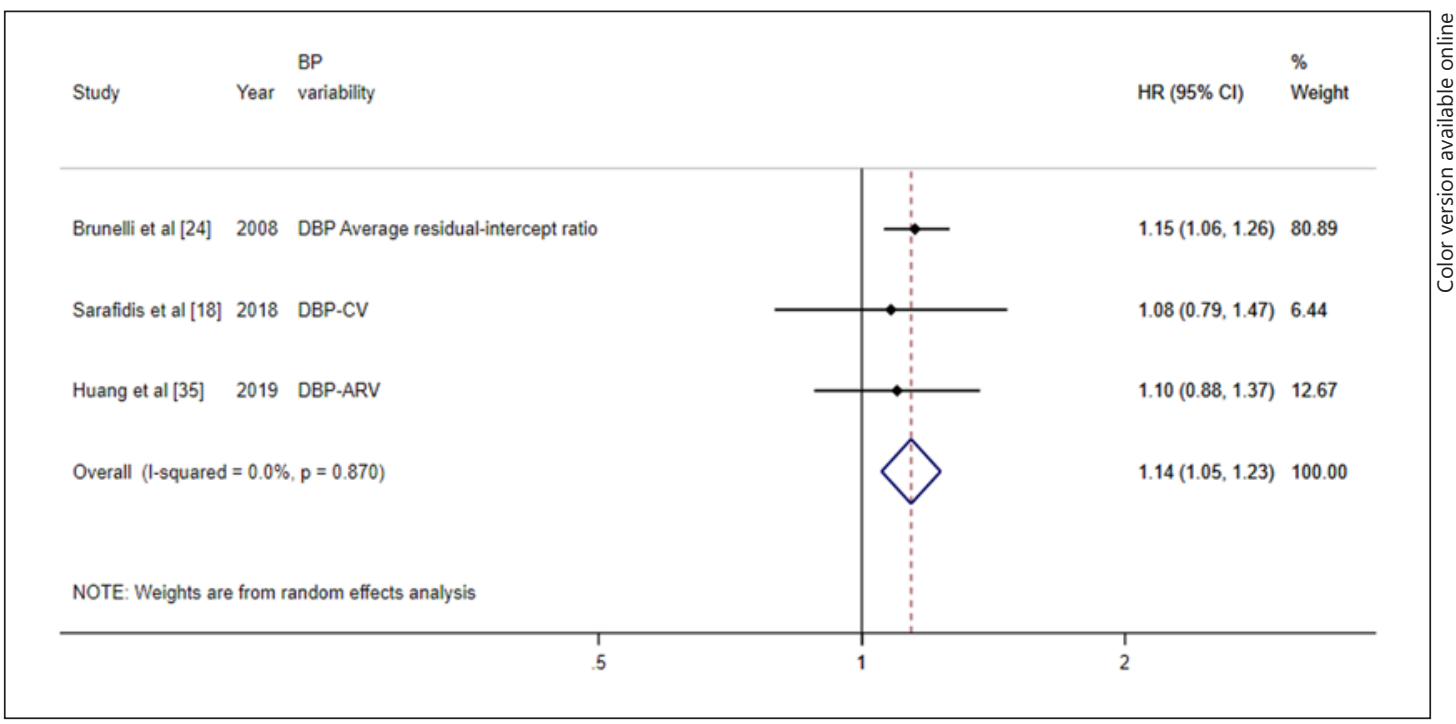

Fig. 5. Forest plot of the relationship between a 1-SD increment in DBPV and all-cause mortality. Boxes represent $\mathrm{HR}$ and lines represent $95 \% \mathrm{CI}$ for individual studies. The diamonds and their width represent pooled HR and the $95 \% \mathrm{CI}$, respectively. DBP, diastolic BP.

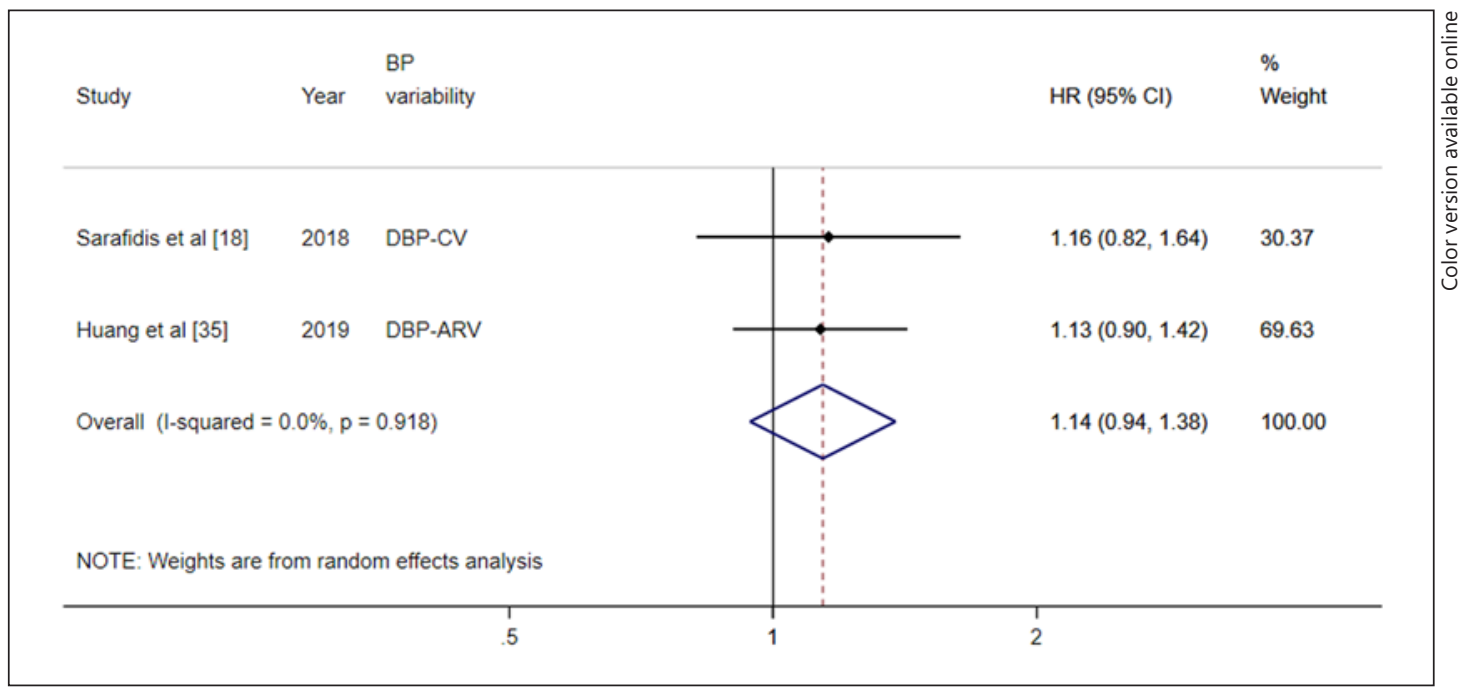

Fig. 6. Forest plot of the relationship between a 1-SD increment in DBPV and cardiovascular mortality. Boxes represent the HR and lines represent $95 \%$ CI for individual studies. The diamonds and their width represent pooled HR and the $95 \% \mathrm{CI}$, respectively. DBP, diastolic BP.

first systematic review and meta-analysis to explore the potential relationship between BPV and adverse outcomes in ESRD patients on HD.

A previous study by Tai et al. [31] reported associations between BPV and mortality and cardiovascular events in patients from multiple populations, including general populations, diabetic patients, and CKD patients who are or are not undergoing dialysis. In general, the results showed that SBPV was associated with higher risks of all-cause mortality (SBP SD for a $1-\mathrm{mm}$ Hg increase: $\mathrm{HR}=1.03 ; 95 \%$ CI 1.02-1.04; SBP CV for a $1 \%$ increase: $\mathrm{HR}=1.04 ; 95 \%$ 
Table 2. Association of all-cause mortality with a 1-SD increase in SBPV in subgroup analyses

\begin{tabular}{|c|c|c|c|}
\hline Variable & Studies, $n$ & HR (95\% CI) & $I^{2}, \%$ \\
\hline \multicolumn{4}{|l|}{ BPV } \\
\hline SD & 4 & $1.27(1.06-1.51)$ & 38.5 \\
\hline $\mathrm{CV}$ & 5 & $1.30(0.99-1.70)$ & 56.4 \\
\hline ARV & 4 & $1.22(0.96-1.55)$ & 66.6 \\
\hline \multicolumn{4}{|l|}{ Study region } \\
\hline Asia & 4 & $1.62(1.02-2.57)$ & 49 \\
\hline Other & 6 & $1.17(1.10-1.24)$ & 58.9 \\
\hline \multicolumn{4}{|l|}{ Study design } \\
\hline Prospective & 3 & $1.24(1.09-1.41)$ & 21 \\
\hline Retrospective & 7 & $1.16(1.07-1.25)$ & 44.7 \\
\hline \multicolumn{4}{|l|}{ Age } \\
\hline$>60$ years & 5 & $1.21(1.10-1.33)$ & 50.4 \\
\hline$\leq 60$ years & 5 & $1.16(1.05-1.28)$ & 44.5 \\
\hline \multicolumn{4}{|l|}{ Women } \\
\hline$>50 \%$ & 2 & $1.80(0.53-6.13)$ & 80.1 \\
\hline$\leq 50 \%$ & 8 & $1.21(1.16-1.25)$ & 0 \\
\hline \multicolumn{4}{|l|}{ Follow-up duration } \\
\hline$>5$ years & 2 & $1.89(0.62-5.80)$ & 75.2 \\
\hline$\leq 5$ years & 8 & $1.18(1.10-1.25)$ & 53.6 \\
\hline \multicolumn{4}{|l|}{ Adjustment factors } \\
\hline Adjusted for IDWG & 4 & $1.21(1.11-1.31)$ & 0 \\
\hline Not adjusted for IDWG & 6 & $1.17(1.08-1.28)$ & 69.4 \\
\hline
\end{tabular}

IDWG, interdialytic weight gain.

CI 1.02-1.06). For cardiovascular mortality, the corresponding HR for SD and CV were 1.10 (95\% CI 1.02-1.17) and 1.01 (95\% CI 0.99-1.03), respectively. The subgroup analysis for allcause mortality in CKD patients included only 2 studies [42, 43], and the combined HR was 1.03 (95\% CI 1.01-1.05). Another systematic review conducted by Stevens et al. [44] included patients not on HD, and it was found that the long-term variability in BP was associated with cardiovascular and mortality outcomes, independently of the mean BP. Compared with previous systematic reviews $[31,44]$, the population in our study was comprised of patients undergoing dialysis, in whom changes in BP are more common than in the general population. Our results showed that a 1-SD increase in SBPV was significantly associated with an $18 \%$ higher risk of all-cause mortality, a 23\% higher risk of cardiovascular mortality, and a $27 \%$ higher risk of cardiovascular events. The results of our study suggest that BPV is a strong predictor of mortality and cardiovascular events among individuals on renal dialysis in addition to the general population. The evidence may offer an important insight into the importance of monitoring the BP fluctuations in patients on dialysis. Clinicians usually emphasized SBPV instead of DBPV. Indeed, the associations with organ damage were usually reported for SBPV $[45,46]$. To the best of our knowledge, no meta-analysis has been done to address the prognostic value of DBPV in CKD patients or dialysis patients. Thus we performed the analyses for DBPV in HD patients. Our study demonstrated that associations were still statistically significant for DBPV and all-cause mortality, cardiovascular mortality, and cardiovascular events $(\mathrm{HR}=1.14 ; 95 \% \mathrm{CI} 1.05-1.23$; $\mathrm{HR}=1.14 ; 95 \% \mathrm{CI} 0.94-1.38$; and $\mathrm{HR}=1.35$; 95\% CI 1.02-1.79, respectively).

Some studies have reported that a higher BPV is related to greater risks of heart disease, stroke, and incidence of CKD [10-13]. Our results imply that BPV is a compelling risk factor for cardiovascular events and mortality among patients undergoing HD. The underlying 


\section{Kidney \\ Blood Pressure \\ Research}

\begin{tabular}{l|l}
\hline Kidney Blood Press Res 2020;45:631-644 \\
\hline DOI: 10.1159/000508979 & $\begin{array}{l}\text { ○ 2020 The Author(s). Published by S. Karger AG, Basel } \\
\text { www.karger.com/kbr }\end{array}$ \\
\hline
\end{tabular}

Li et al.: BPV Predicts Adverse Outcomes in Patients on Dialysis

mechanisms that contribute to the relationship in patients with dialysis are complex and poorly understood. We discussed several possible explanations. Patients on maintenance HD are prone to wide BP fluctuations. Increased BPV might be a manifestation of extracellular fluid volume status. The volume status changes as body fluids are removed over several hours during HD, and fluids are progressively retained during the interdialytic interval. A greater interdialytic weight gain or ultrafiltration volume was associated with increased BPV [47, 48]. Dialysis patients have poor volume control with excessive interdialytic weight gain and higher ultrafiltration rates or volumes during an HD session, leading to increased risks of mortality and cardiovascular morbidity [49]. Our subgroup analysis showed that additional adjustments for interdialytic weight gain or ultrafiltration volume did not change the statistical difference of the results. The results indicated that the underlying mechanism might be independent of interdialytic weight gain or ultrafiltration volume.

Apart from volume-related influences, various factors, such as arterial rigidity, antihypertensive medications, and the sympathetic nervous system, might also facilitate amplification of the BPV [9]. Greater BPV is associated with arterial stiffness [50], which is a very common alteration in CKD patients. Increased arterial stiffness causes left ventricular hypertrophy and reduces myocardial perfusion, which is related to cardiovascular morbidity and mortality [51]. SBPV was reported to be associated with endothelial dysfunction [52]. Greater BPV may induce endothelial inflammation and produce vascular damage. Endothelial dysfunction was related to an increased risk of cardiovascular mortality [53]. The role of the sympathetic nervous system was examined in animal experiments. In rats, sinoaortic denervation increased BPV independently of the mean BP. These rats develop left ventricular hypertrophy and renal sclerosis [54]. Thus, increased BPV may be a marker of underlying autonomic dysfunction.

Various BPV metrics have been applied across studies to describe BP fluctuations. The $\mathrm{SD}$ is a classic metric that is used to evaluate variability. It is easy to calculate, and it is often strongly correlated with the mean BP. CV, VIM, and ARV are other commonly used BPV metrics. However, VIM and ARV, despite advantages for reflecting fluctuations in BP, require complex calculations and have limited clinical application $[14,55]$. CV is a commonly reported BPV metric; we preferred this metric because it accounted for the mean BP [9]. BPV might be difficult to assess in clinical practice, as it requires multiple BP measurements and calculations. However, it may be important as a patient with a high BPV is at a greater risk even when the mean BP is within the normal range.

Our results may help clinicians gain a better understanding of the impact of BPV in HD patients. However, our study had several limitations. First, moderate heterogeneity was present in the analyses of the associations between SBPV and the outcomes. We performed meta-regression and subgroup analyses to identify potential sources of heterogeneity, but heterogeneity remained after these analyses. Because all included studies were observational, this would lead to bias and cofounders for which it is difficult to adjust. Second, most studies reported SBPV and adverse health outcomes. SBP was reported to cause greater risks than DBP [56]. Our study indicated that both SBPV and DBPV have similar predictive roles in mortality and cardiovascular outcomes. However, there was less data on DBPV, and the conclusion regarding the role of DBPV was weak because of the small sample size. Third, no studies reported associations between BPV and mortality or cardiovascular risk in patients on PD. It is still unclear whether BPV has the same predictive role as in HD patients. Future prospective studies need to address this question. Fourth, the therapeutic effect of reducing $\mathrm{BPV}$ is still uncertain. There is no randomized data proving that reducing BPV would improve clinical outcomes. However, our results strengthen the link between BPV and adverse outcomes among HD patients. It is reasonable to believe that strategies (such as adequate antihypertensives treatment, a slower ultrafiltration rate, and minimization of interdialytic 
weight gain) to reduce BPV may be beneficial for this vulnerable group of patients. Still, future randomized clinical trials are needed to verify the hypotheses.

In conclusion, the findings of this systematic review and meta-analysis suggest that increased SBPV and DBPV are associated with the risks of all-cause mortality, cardiovascular mortality, and cardiovascular events. Given the presence of moderate heterogeneity, further interventional studies are needed to verify our conclusions in real time and in real patients. Our results indicate that identifying those with a high BPV is important, and preventing large fluctuations in BP may be an additional therapeutic target in BP management in HD patients.

\section{Statement of Ethics}

The authors have no ethical conflicts to declare.

\section{Conflict of Interest Statement}

The authors have no conflict of interests to declare.

\section{Funding Sources}

This study was financially supported by the National Youth Science Foundation of China (No.81600536), the Natural Science Foundation of Hunan Province of China (No. 2018JJ3833), and China's Xiangya medical big data project (No. 33020125032).

\section{Author Contributions}

W.C.: conceptualization. H.L. and J.X.: data curation. J.X. and W.C.: formal analysis and methodology. Q.Z.: supervision. H.L., X.L., and P.Z.: writing of the original draft. W.C. and W.D.: writing - review and editing.

\section{References}

1 Held PJ, Port FK, Wolfe RA, Stannard DC, Carroll CE, Daugirdas JT, et al. The dose of hemodialysis and patient mortality. Kidney Int. 1996 Aug;50(2):550-6.

2 Saran R, Robinson B, Abbott KC, Agodoa LY, Bhave N, Bragg-Gresham J, et al. US renal data system 2017 annual data report: epidemiology of kidney disease in the United States. Am J Kidney Dis. 2018 Mar; 71(3 Suppl 1):A7.

3 Song KK, Zhao DL, Wang YD, Wang Y, Sun XF, Miao LN, et al. Analysis of factors associated with death in maintenance hemodialysis patients: a multicenter study in China. Chin Med J (Engl). 2017 Apr;130(8):885-91.

4 Zhang F, Liu H, Gong X, Liu F, Peng Y, Cheng M, et al. Risk factors for mortality in Chinese patients on continuous ambulatory peritoneal dialysis. Perit Dial Int. 2015 Mar-Apr;35(2):199-205.

5 Chirakarnjanakorn S, Navaneethan SD, Francis GS, Tang WH. Cardiovascular impact in patients undergoing maintenance hemodialysis: clinical management considerations. Int J Cardiol. 2017 Apr;232:12-23.

6 Mancia G, Fagard R, Narkiewicz K, Redón J, Zanchetti A, Böhm M, et al.; Task Force Members. 2013 ESH/ESC Guidelines for the management of arterial hypertension: the Task Force for the management of arterial hypertension of the European Society of Hypertension (ESH) and of the European Society of Cardiology (ESC). J Hypertens. 2013 Jul;31(7):1281-357.

7 Heerspink HJ, Ninomiya T, Zoungas S, de Zeeuw D, Grobbee DE, Jardine MJ, et al. Effect of lowering blood pressure on cardiovascular events and mortality in patients on dialysis: a systematic review and meta-analysis of randomised controlled trials. Lancet. 2009 Mar;373(9668):1009-15. 
8 Sogunuru GP, Kario K, Shin J, Chen CH, Buranakitjaroen P, Chia YC, et al.; HOPE Asia Network. Morning surge in blood pressure and blood pressure variability in Asia: Evidence and statement from the HOPE Asia Network. J Clin Hypertens (Greenwich). 2019 Feb;21(2):324-34.

9 Parati G, Ochoa JE, Lombardi C, Bilo G. Assessment and management of blood-pressure variability. Nat Rev Cardiol. 2013 Mar;10(3):143-55.

10 Yu ZB, Wang JB, Li D, Chen XY, Lin HB, Chen K. Prognostic value of visit-to-visit systolic blood pressure variability related to diabetic kidney disease among patients with type 2 diabetes. J Hypertens. 2019 Jul;37(7): 1411-8.

11 Wang H, Li M, Xie SH, Oyang YT, Yin M, Bao B, et al. Visit-to-visit Systolic Blood Pressure Variability and Stroke Risk: A Systematic Review and Meta-analysis. Curr Med Sci. 2019 Oct;39(5):741-7.

12 Viazzi F, Bonino B, Mirijello A, Fioretto P, Giorda C, Ceriello A, et al.; AMD-Annals Study Group. Long-term blood pressure variability and development of chronic kidney disease in type 2 diabetes. J Hypertens. 2019 Apr; 37(4):805-13.

13 Wang J, Shi X, Ma C, Zheng H, Xiao J, Bian H, et al. Visit-to-visit blood pressure variability is a risk factor for allcause mortality and cardiovascular disease: a systematic review and meta-analysis. J Hypertens. 2017 Jan; 35(1):10-7.

14 Rothwell PM, Howard SC, Dolan E, O’Brien E, Dobson JE, Dahlöf B, et al. Prognostic significance of visit-to-visit variability, maximum systolic blood pressure, and episodic hypertension. Lancet. 2010 Mar;375(9718):895-905.

15 Agarwal R. Blood pressure and mortality among hemodialysis patients. Hypertension. 2010 Mar;55(3):762-8.

16 Georgianos PI, Agarwal R. Blood Pressure and Mortality in Long-Term Hemodialysis-Time to Move Forward. Am J Hypertens. 2017 Mar;30(3):211-22.

17 Alborzi P, Patel N, Agarwal R. Home blood pressures are of greater prognostic value than hemodialysis unit recordings. Clin J Am Soc Nephrol. 2007 Nov;2(6):1228-34.

18 Sarafidis PA, Loutradis C, Karpetas A, Tzanis G, Bikos A, Raptis V, et al. The association of interdialytic blood pressure variability with cardiovascular events and all-cause mortality in haemodialysis patients. Nephrol Dial Transplant. 2019 Mar;34(3):515-23.

19 Liao R, Li J, Lin L, Sun S, Wang L, Xiong Y, et al. The Association between Long- and Intra-Dialytic Blood Pressure Variability with All-Cause Mortality in Hemodialysis Patients. Blood Purif. 2019;48(1):43-50.

20 Liao R, Li J, Xiong Y, Lin L, Wang L, Sun S, et al. Association of Peridialysis Blood Pressure and Its Variability with Cardiovascular Events in Hemodialysis Patients. Kidney Blood Press Res. 2018;43(4):1352-62.

21 Feng Y, Li Z, Liu J, Sun F, Ma L, Shen Y, et al. Association of short-term blood pressure variability with cardiovascular mortality among incident hemodialysis patients. Ren Fail. 2018 Nov;40(1):259-64.

22 Kim HY, Kang YU, Kim CS, Choi JS, Bae EH, Ma SK, et al. Association of age and BP variability with long-term mortality in hemodialysis patients. Kidney Blood Press Res. 2013;38(2-3):172-80.

23 Rossignol P, Cridlig J, Lehert P, Kessler M, Zannad F. Visit-to-visit blood pressure variability is a strong predictor of cardiovascular events in hemodialysis: insights from FOSIDIAL. Hypertension. 2012 Aug;60(2): 339-46.

24 Brunelli SM, Thadhani RI, Lynch KE, Ankers ED, Joffe MM, Boston R, et al. Association between long-term blood pressure variability and mortality among incident hemodialysis patients. Am J Kidney Dis. 2008 Oct;52(4): 716-26.

25 Tozawa M, Iseki K, Yoshi S, Fukiyama K. Blood pressure variability as an adverse prognostic risk factor in endstage renal disease. Nephrol Dial Transplant. 1999 Aug;14(8):1976-81.

26 Stroup DF, Berlin JA, Morton SC, Olkin I, Williamson GD, Rennie D, et al. Meta-analysis of observational studies in epidemiology: a proposal for reporting. Meta-analysis Of Observational Studies in Epidemiology (MOOSE) group. JAMA. 2000 Apr;283(15):2008-12.

27 Chandler J, Cumpston M, Li T, Page MJ, Welch VA. Cochrane handbook for systematic reviews of interventions. Hoboken: Wiley; 2019.

28 Moher D, Liberati A, Tetzlaff J, Altman DG; PRISMA Group. Preferred reporting items for systematic reviews and meta-analyses: the PRISMA statement. J Clin Epidemiol. 2009 Oct;62(10):1006-12.

29 Wells G, Shea B, O'connell D, Peterson J, Welch V, Losos M, et al. The Newcastle-Ottawa Scale (NOS) for assessing the quality of nonrandomised studies in meta-analyses. Ottawa: Ottawa Hospital Research Institute, 2014.

30 Chiriacò M, Pateras K, Virdis A, Charakida M, Kyriakopoulou D, Nannipieri M, et al. Association between blood pressure variability, cardiovascular disease and mortality in type 2 diabetes: A systematic review and metaanalysis. Diabetes Obes Metab. 2019;21(12):2587-98.

31 Tai C, Sun Y, Dai N, Xu D, Chen W, Wang J, et al. Prognostic significance of visit-to-visit systolic blood pressure variability: a meta-analysis of 77,299 patients. J Clin Hypertens (Greenwich). 2015 Feb;17(2):107-15.

32 Danesh J, Collins R, Appleby P, Peto R. Association of fibrinogen, C-reactive protein, albumin, or leukocyte count with coronary heart disease: meta-analyses of prospective studies. JAMA. 1998 May;279(18):1477-82.

33 Higgins JP, Thompson SG. Quantifying heterogeneity in a meta-analysis. Stat Med. 2002 Jun;21(11):1539-58.

34 Begg CB, Mazumdar M. Operating characteristics of a rank correlation test for publication bias. Biometrics. 1994 Dec;50(4):1088-101.

35 Huang JT, Cheng HM, Yu WC, Lin YP, Sung SH, Chen CH. Increased Nighttime Pulse Pressure Variability but Not Ambulatory Blood Pressure Levels Predicts 14-Year All-Cause Mortality in Patients on Hemodialysis. Hypertension. 2019 Sep;74(3):660-8. 
36 Amari Y, Morimoto S, Iida T, Yurugi T, Oyama Y, Aoyama N, et al. Characteristics of visit-to-visit blood pressure variability in hemodialysis patients. Hypertens Res. 2019 Jul;42(7):1036-1048.

37 Wang Y, Qin Y, Fan X, Cai J, Ye W, Xia J, et al. Variability in Predialysis Systolic Blood Pressure and Long-Term Outcomes in Hemodialysis Patients. Kidney Blood Press Res. 2018;43(1):115-24.

38 Shafi T, Sozio SM, Bandeen-Roche KJ, Ephraim PL, Luly JR, St Peter WL, et al.; DEcIDE Network Patient Outcomes in End Stage Renal Disease Study Investigators. Predialysis systolic BP variability and outcomes in hemodialysis patients. J Am Soc Nephrol. 2014 Apr;25(4):799-809.

39 Selvarajah V, Pasea L, Ojha S, Wilkinson IB, Tomlinson LA. Pre-dialysis systolic blood pressure-variability is independently associated with all-cause mortality in incident haemodialysis patients. PLoS One. 2014 Jan; 9(1):e86514.

40 Chang TI, Flythe JE, Brunelli SM, Muntner P, Greene T, Cheung AK, et al. Visit-to-visit systolic blood pressure variability and outcomes in hemodialysis. J Hum Hypertens. 2014 Jan;28(1):18-24.

41 Flythe JE, Inrig JK, Shafi T, Chang TI, Cape K, Dinesh K, et al. Association of intradialytic blood pressure variability with increased all-cause and cardiovascular mortality in patients treated with long-term hemodialysis. Am J Kidney Dis. 2013 Jun;61(6):966-74.

42 Di Iorio B, Di Micco L, Torraca S, Sirico ML, Guastaferro P, Chiuchiolo L, et al. Variability of blood pressure in dialysis patients: a new marker of cardiovascular risk. J Nephrol. 2013 Jan-Feb;26(1):173-82.

43 Mallamaci F, Minutolo R, Leonardis D, D’Arrigo G, Tripepi G, Rapisarda F, et al. Long-term visit-to-visit office blood pressure variability increases the risk of adverse cardiovascular outcomes in patients with chronic kidney disease. Kidney Int. 2013 Aug;84(2):381-9.

44 Stevens SL, Wood S, Koshiaris C, Law K, Glasziou P, Stevens RJ, et al. Blood pressure variability and cardiovascular disease: systematic review and meta-analysis. BMJ. 2016 Aug;354:i4098.

45 Tatasciore A, Renda G, Zimarino M, Soccio M, Bilo G, Parati G, et al. Awake systolic blood pressure variability correlates with target-organ damage in hypertensive subjects. Hypertension. 2007 Aug;50(2):325-32.

46 Leoncini G, Viazzi F, Storace G, Deferrari G, Pontremoli R. Blood pressure variability and multiple organ damage in primary hypertension. J Hum Hypertens. 2013 Nov;27(11):663-70.

47 Inrig JK, Patel UD, Gillespie BS, Hasselblad V, Himmelfarb J, Reddan D, et al. Relationship between interdialytic weight gain and blood pressure among prevalent hemodialysis patients. Am J Kidney Dis. 2007 Jul;50(1): 108-18.

48 Van Buren PN, Shastri S, Inrig JK. New perspectives on blood pressure variability in hemodialysis patients. Am J Kidney Dis. 2012 Mar;59(3):333-5.

49 Ok E, Asci G, Chazot C, Ozkahya M, Mees EJ. Controversies and problems of volume control and hypertension in haemodialysis. Lancet. 2016 Jul;388(10041):285-93.

50 Kotsis V, Stabouli S, Karafillis I, Papakatsika S, Rizos Z, Miyakis S, et al. Arterial stiffness and $24 \mathrm{~h}$ ambulatory blood pressure monitoring in young healthy volunteers: the early vascular ageing Aristotle University Thessaloniki Study (EVA-ARIS Study). Atherosclerosis. 2011 Nov;219(1):194-9.

51 Gusbeth-Tatomir P, Covic A. Causes and consequences of increased arterial stiffness in chronic kidney disease patients. Kidney Blood Press Res. 2007;30(2):97-107.

52 Diaz KM, Veerabhadrappa P, Kashem MA, Feairheller DL, Sturgeon KM, Williamson ST, et al. Relationship of visit-to-visit and ambulatory blood pressure variability to vascular function in African Americans. Hypertens Res. 2012;35(1):55-61.

53 Stam F, van Guldener C, Becker A, Dekker JM, Heine RJ, Bouter LM, et al. Endothelial dysfunction contributes to renal function-associated cardiovascular mortality in a population with mild renal insufficiency: the Hoorn study. J Am Soc Nephrol. 2006 Feb;17(2):537-45.

54 Miao CY, Xie HH, Zhan LS, Su DF. Blood pressure variability is more important than blood pressure level in determination of end-organ damage in rats. J Hypertens. 2006 Jun;24(6):1125-35.

55 Mena L, Pintos S, Queipo NV, Aizpúrua JA, Maestre G, Sulbarán T. A reliable index for the prognostic significance of blood pressure variability. J Hypertens. 2005 Mar;23(3):505-11.

56 Stamler J, Stamler R, Neaton JD. Blood pressure, systolic and diastolic, and cardiovascular risks. US population data. Arch Intern Med. 1993 Mar;153(5):598-615. 